\title{
Generation of monoclonal antibodies against native viral proteins using antigen- expressing mammalian cells for mouse immunization
}

\author{
Natalie Rose ${ }^{1,2}$, Carlos Augusto Pinho-Nascimento ${ }^{3}$, Alessia Ruggieri ${ }^{4}$, Paola Favuzza ${ }^{1,2}$, Marco Tamborrini ${ }^{1,2}$, \\ Hanna Roth ${ }^{4}$, Marcia Terezinha Baroni de Moraes ${ }^{5}$, Hugues Matile ${ }^{1,2}$, Thomas Jänisch ${ }^{6}$, Gerd Pluschke ${ }^{1,2}$ \\ and Katharina Röltgen ${ }^{1,2^{*}}$
}

\begin{abstract}
Background: Due to their rising incidence and progressive geographical spread, infections with mosquito-borne viruses, such as dengue (DENV), chikungunya and zika virus, have developed into major public health challenges. Since all of these viruses may cause similar symptoms and can occur in concurrent epidemics, tools for their differential diagnosis and epidemiological monitoring are of urgent need.

Results: Here we report the application of a novel strategy to rapidly generate monoclonal antibodies (mAbs) against native viral antigens, exemplified for the DENV nonstructural glycoprotein 1 (NS1). The described system is based on the immunization of mice with transfected mammalian cells expressing the target antigens in multiple displays on their cell surface and thereby presenting them efficiently to the host immune system in their native conformation. By applying this cell-based approach to the DENV NS1 protein of serotypes 1 (D1NS1) and 4 (D4NS1), we were able to rapidly generate panels of DENV NS1 serotype cross-reactive, as well as D1NS1- and D4NS1 serotype-specific mAbs. Our data show that the generated mAbs were capable of recognizing the endogenous NS1 protein in DENV-containing biological samples.

Conclusion: The use of this novel immunization strategy, allows for a fast and efficient generation of hybridoma cell lines, producing mAbs against native viral antigens. Envisaged applications of the mAbs include the development of test platforms enabling a differentiation of the DENV serotypes and high resolution immunotyping for epidemiological studies.
\end{abstract}

Keywords: Dengue virus, NS1 protein, Mouse immunization, HEK cells, Transfection, Hybridoma technology, Monoclonal antibodies

\section{Background}

The past decades have witnessed a dramatic upsurge or emergence of arboviral diseases [1], including dengue $[2,3]$, chikungunya [4], and zika [5-7]. Since transmission of these viruses occurs through Aedes aegypti - one of the most widespread mosquito species globally - the potential

\footnotetext{
* Correspondence: Katharina.roeltgen@unibas.ch

${ }^{1}$ Swiss Tropical and Public Health Institute, Molecular Immunology, Basel, Switzerland

${ }^{2}$ University of Basel, Basel, Switzerland

Full list of author information is available at the end of the article
}

for major and possibly concurrent epidemics of these viruses and other as yet unknown mosquito-borne viruses that might emerge, is overwhelming, and emphasizes the pressing need to develop vaccines and antiviral therapeutics [5]. Moreover, diagnostic tools to reliably distinguish between the various viral infections that often lead to similar clinical symptoms [8], but necessitate distinct management, are urgently required. Dengue is considered the currently most important arboviral disease [3], with an estimated 390 million cases annually [9]. Infections are caused by one of at least four antigenically distinct 
serotypes (DENV1-4) that vary by 25 to $40 \%$ at the amino acid level [10]. It has been reported that secondary infection with a heterologous serotype is associated with an increased relative risk of severe disease [10, 11]. Adequate management of severe dengue cases can greatly reduce the death rate. To date, diagnosis of the infecting serotype is of epidemiological interest, and in the future could potentially be relevant for prognosis in individual patients. Moreover, the canonical DENV serotypes appear to be antigenically more diverse than previously assumed, requiring more detailed studies of the relevance of individual antigenic determinants for clinical severity, epidemic magnitude, and DENV evolution [12].

Ever since the development of the B cell hybridoma technology in 1975 [13], the application of monoclonal antibodies (mAbs) as tools in the development of vaccines, diagnostics and therapeutics, and as general research tools has augmented [14-16]. MAbs provide a number of unique properties including the ability to bind specifically and with high affinity to almost any molecular structure as well as their availability in unlimited quantities as homogeneous reagents. Prerequisite for the generation of mAbs by the $\mathrm{B}$ cell hybridoma technology is the immunization of animals - most commonly mice - with the specific target antigen. In the case of proteins as target structures, immunization of mice has traditionally been accomplished by using recombinantly expressed and purified proteins, an often time-consuming and tedious endeavor. Due to their clear advantage in terms of yield and cost, simple prokaryotic expression systems, particularly Escherichia coli, or lower eukaryotic hosts, such as yeast, have long dominated the field of recombinant protein expression [17]. However, mammalian expression systems that are capable of providing native protein folding, and natural posttranslational modifications, have been increasingly used in the past years to reliably express proteins in their native conformation [18].

In order to meet the requirements for mAbs against viral proteins for various public health and research applications, we evaluated here a fast and highly efficient strategy of mouse immunization with native viral antigens using mammalian cells that express the recombinant proteins on their surface. This system is based on the transfection of the human embryonic kidney (HEK) suspension cell line $293 \mathrm{~F}$ with a plasmid vector encoding the protein of interest as fusion protein containing a transmembrane domain, and thereby enabling the presentation of large amounts of the chimeric membrane-bound target antigen on the HEK cell surface in a native conformation to the immune system of animals immunized with the transfected cells. The DENV genome encodes for three structural (C, prM and E) and seven nonstructural (NS1, NS2A, NS2B, NS3, NS4A, NS4B and NS5) proteins [19]. We have chosen the DENV NS1 protein as recombinant target antigen in this study, since NS1 is an important biomarker for early diagnosis of dengue infections and has also been intensively investigated as a potential target for vaccines and antibody-based antiviral therapeutics. Depending on its glycosylation status, NS1 has a molecular weight of $46-55 \mathrm{kDa}$. The protein exists in multiple oligomeric forms and occurs as intracellular membraneassociated form, on the cell surface, or as a soluble, secreted lipoparticle [20] that can be detected in the serum of dengue patients during the acute phase of the disease [21].

The specific objectives of the present study were i) to prove the suitability of the applied immunization strategy for viral proteins, ii) to generate mAbs specifically recognizing the D1NS1 and D4NS1 proteins in their native conformation, and iii) to show that these mAbs can be used to detect the endogenous protein in DENV-containing biological samples.

\section{Methods \\ Ethics statement}

Immunization of Naval Medical Research Institute (NMRI) mice was performed in strict accordance with the rules and regulations for the protection of animal rights of the Swiss Federal Food Safety and Veterinary Office. Experiments were approved by the animal welfare committee of the Canton of Basel (authorization number 2375).

\section{Construction and amplification of plasmids containing DENV NS1 protein-encoding sequences}

An expression plasmid pcDNA3.1 (Invitrogen, San Diego, CA) was used that has been modified to supply inserted genes with the secretion signal sequence of bee venom mellitin (BVM), a FLAG-tag, a trans-membrane (TM) domain and a hexa-His tag [22] to enable the expression of specific proteins on the surface of mammalian cells transfected with the plasmid. In addition to the DENV D1NS1 and D4NS1 protein used for immunization of mice and generation of mAbs, we also expressed the D2NS1 and D3NS1 proteins for analyses of the DENV serotype-specificity of the generated mAbs. The D1NS1 D4NS1-encoding sequences (amino acid sequences are provided in Additional file 1: Figure S1), were cloned with restriction sites NheI and NotI into the modified pcDNA3.1 plasmid. For amplification of the constructed pcDNA3.1_BVM_D1NS1_FLAG_TM_HIS, pcDNA3.1_ BVM_D2NS1_FLAG_TM_HIS, pcDNA3.1_BVM_D3NS1_ FLAG_TM_HIS and pcDNA3.1_BVM_D4NS1_FLAG_ TM_HIS plasmids, chemically competent E. coli One Shot Top10 cells (Invitrogen, San Diego, CA) were transformed according to the manufacturer's instructions and grown in LB medium containing $100 \mu \mathrm{g} / \mathrm{ml}$ ampicillin. DNA was extracted and purified using the NucleoBond Xtra Maxi Plus 
plasmid DNA purification kit (Macherey-Nagel, Düren, Germany).

\section{Transfection of HEK $293 \mathrm{~F}$ cells}

The HEK cell line 293F was grown in FreeStyle 293 Expression Medium (Gibco, Grand Island, NY) in $125 \mathrm{ml}$ disposable polycarbonate Erlenmeyer flasks (Corning, Oneonta, NY). Cells were maintained in a humidified incubator at $37{ }^{\circ} \mathrm{C}$ in $5 \% \mathrm{CO}_{2}$ on a platform shaker with rotation at $150 \mathrm{rpm}$ and were passaged when the concentration of viable cells reached $2 \times 10^{6}$ cells per ml. For the transfections, $50 \mu \mathrm{g}$ of plasmid DNA and $150 \mu \mathrm{l}$ of Lipofectamine 2000 Reagent (Invitrogen, Carlsbad, CA) were diluted each in $2.5 \mathrm{ml}$ FreeStyle medium. DNA was added to the Lipofectamine Reagent and incubated for $5 \mathrm{~min}$ at room temperature. The mix was then added to $45 \mathrm{ml}$ of HEK cells diluted to $10^{6}$ cells per ml and transfected cells were cultured as described above. After $48 \mathrm{~h}$, transfected cells (D1NS1-HEK - D4NS1-HEK) were harvested. Levels of NS1 expression were assessed by Western blot analysis and immunofluorescence assay (IFA). Aliquots of $6 \times 10^{6}$ transfected HEK cells each were stored in freezing medium (50 \% fetal bovine serum, $40 \%$ Freestyle medium and $10 \%$ dimethylsulfoxid) in order to preserve the viability of the transfected cells at $-80{ }^{\circ} \mathrm{C}$ until further use.

\section{Generation of mAbs against the D1NS1 and D4NS1 proteins}

Stored aliquots of $6 \times 10^{6}$ transfected D1NS1-HEK or D4NS1-HEK cells were thawed, washed and re-suspended in $0.9 \%$ sodium chloride. Immunization of NMRI mice was conducted by intravenous injections of $10^{6}$ transfected HEK cells per dose in two cycles of four consecutive days with a break between cycles of 1 week. Mice with high anti-DENV NS1 IgG titers as determined by IFA of mouse sera on transfected and untransfected HEK cells and by ELISA of mouse sera on recombinant DENV NS1 (recD1NS1 and recD4NS1) proteins (AbD Serotec, Puchheim, Germany) were selected. The recombinant D1NS1 (sequence strain Nauru/Western Pacific/1974) and D4NS1 (sequence strain Dominica/814669/1981) proteins purchased from AbD Serotec are purified recombinant proteins expressed in HEK293 cells and presented in their native folded state with post-translational modifications. Selected mice received a final boost of $10^{6}$ transfected HEK cells each on two consecutive days. Two days after the last injections, selected mice were sacrificed and their spleens were aseptically removed. Mouse spleen cells and PAI myeloma cells were fused to generate antibody-producing hybridoma cell lines as described [23].

\section{DENV production}

DENV serotype 1 (strain Hawaii), DENV serotype 3 (strain H87), and DENV serotype 4 (strain H241) virus stocks were prepared by virus amplification in VeroE6 cells. Virus stock titers were determined by plaque assay. In brief, VeroE6 cells were infected with serial dilutions of virus supernatants. Two hours post-infection the inoculum was replaced by serum-free MEM medium (Life Technologies, Darmstadt, Germany) containing $1.5 \%$ carboxymethyl cellulose (Sigma-Aldrich, Taufkirchen, Germany). Five days post-infection, cells were fixed by addition of formaldehyde to a final concentration of $5 \%$. Cells were stained with crystal violet solution (1\% crystal violet, $10 \%$ ethanol in $\mathrm{H}_{2} \mathrm{O}$ ) for $30 \mathrm{~min}$ at room temperature and rinsed extensively with $\mathrm{H}_{2} \mathrm{O}$. Infectious titers were calculated considering the corresponding dilution factor. DENV serotype 2 (strain New Guinea C) virus stock was produced by electroporation of BHK-21 cells with in vitro transcripts and further amplification of the BHK-21 supernatant in VeroE6 cells as described above. Virus stock titer was determined by plaque assay and cells were fixed 7 days post infection.

\section{Western blot analysis with HEK cell and VeroE6 cell lysates}

In order to prepare HEK cell protein lysates, aliquots of $6 \times 10^{6}$ transfected or untransfected (negative control) HEK cells were thawed, washed and re-suspended in $600 \mu \mathrm{l}$ of RIPA buffer containing $20 \mathrm{mM}$ Tris $\mathrm{HCl}$, $137 \mathrm{mM} \mathrm{NaCl}, 10$ \% glycerol, 1 \% NP-40, $0.25 \%$ sodium deoxycholate and a protease inhibitor cocktail (cOmplete Mini; Roche, Mannheim, Germany). After incubation for $30 \mathrm{~min}$ on ice, the mix was centrifuged at $10^{\prime} 000 \mathrm{rpm}$ for $10 \mathrm{~min}$ and the supernatant was stored at $-20^{\circ} \mathrm{C}$.

For VeroE6 cell lysates, $10^{6}$ cells were infected with a multiplicity of infection of 10 for $36 \mathrm{~h}$ (DENV serotypes 1, 2 and 4) and $48 \mathrm{~h}$ (DENV serotype 3). Cells were lysed with $100 \mu \mathrm{l}$ ice-cold lysis buffer $(50 \mathrm{mM}$ Tris $\mathrm{HCl}$ $\mathrm{pH}$ 7.4, $150 \mathrm{mM} \mathrm{NaCl}, 1$ \% Triton X-100, $60 \mathrm{mM} \beta$-glycerophosphate, $15 \mathrm{mM}$ 4-nitrophenylphosphate, $1 \mathrm{mM}$ sodium orthovanadate, $1 \mathrm{mM}$ sodium fluoride, and protease inhibitor cocktail).

For SDS-PAGE, $2 \mu \mathrm{g}$ of HEK cell lysates per lane were separated on NuPAGE Novex 4-12 \% Bis-Tris Gels (Invitrogen, Carlsbad, CA) with addition of NuPage Reducing Agent (Invitrogen, Carlsbad, CA) and heating of the samples for $5 \mathrm{~min}$ at $95{ }^{\circ} \mathrm{C}$ (reducing conditions). Lysates of DENV-infected VeroE6 cells were separated on NuPAGE Novex 4-12 \% Bis-Tris Zoom Gels under nonreducing conditions (without addition of reducing agent and without heating of samples). After electrophoresis, protein bands were either visualized directly with aquastaining or were transferred onto nitrocellulose membranes for Western blot analysis using an iBlot Gel Transfer 
Device (Invitrogen). Membranes were blocked with $5 \%$ non-fat dry milk in PBS containing $0.1 \%$ Tween 20 and cut into strips, if required. Membranes or membrane strips were then incubated with anti-hexa-His tag, antitubulin or anti-DENV NS1 mAbs, washed with PBS containing $0.1 \%$ Tween 20 and thereafter incubated with horseradish peroxidase-conjugated goat anti-mouse IgG antibodies ( $\gamma$-chain specific; Southern Biotech, Birmingham, $\mathrm{AL}$ ). After a second washing step, bands were visualized by chemiluminescence using ECL Western Blotting substrate (Thermo Scientific, Rockford, IL).

\section{Immunofluorescence staining of HEK cells}

For IFA, an aliquot of $6 \times 10^{6}$ transfected HEK cells was thawed, washed and re-suspended in $600 \mu \mathrm{l}$ of PBS. Each well of 12-well multi-test glass slides (MP Biomedicals, Inc., Illkirch, France) was coated with $2 \mu \mathrm{l}$ of the cell suspension. After air-drying under a sterile hood, cells were fixed in methanol for $15 \mathrm{~min}$. Dried wells were then incubated with either hybridoma cell culture supernatant or purified mAbs diluted in PBS containing $0.5 \%$ BSA for $20 \mathrm{~min}$ in a wet chamber at $37{ }^{\circ} \mathrm{C}$. Following rinsing, washing twice for $5 \mathrm{~min}$ in $\mathrm{PBS}$ containing $0.05 \%$ Tween 20 and rinsing in distilled $\mathrm{H}_{2} \mathrm{O}$, dried wells were incubated with Alexa568-conjugated goat anti-mouse IgG antibodies (KPL, Gaithersburg, MD) diluted in PBS containing $0.5 \% \mathrm{BSA}$ for $20 \mathrm{~min}$ in a wet chamber at $37{ }^{\circ} \mathrm{C}$. After washing as described, dried slides were mounted with ProLong Gold antifade reagent with DAPI (Life technologies, Eugene, OR) and covered with a cover slip. Analyses were performed on a Leica CTR500 fluorescence microscope and images were taken with a Leica DFC345 FX digital camera (Leica Microsystems, Heerbrugg, Switzerland).

\section{Conventional ELISA on recombinant NS1 proteins}

96-well Nunc-Immuno Maxisorp plates (Thermo Scientific, Roskilde, Denmark) were coated overnight at $4{ }^{\circ} \mathrm{C}$ in a wet chamber with $50 \mu \mathrm{l}$ PBS containing recombinant DENV NS1 (recD1NS1 and recD4NS1) protein (AbD Serotec, Puchheim, Germany) at a concentration of $1 \mu \mathrm{g} / \mathrm{ml}$ or an unrelated hexa-His tagged protein (MUL_3720 [24]) serving as a control. After washing with distilled $\mathrm{H}_{2} \mathrm{O}$ containing $0.01 \%$ Tween 20, wells were blocked with $5 \%$ non-fat dry milk in PBS for $1 \mathrm{~h}$ at $37^{\circ} \mathrm{C}$. Subsequently, wells were incubated with mouse antisera or hybridoma cell culture supernatant diluted in PBS containing $0.5 \%$ milk and $0.05 \%$ Tween 20 for $1 \mathrm{~h}$ at $37{ }^{\circ} \mathrm{C}$. After washing as described, horseradish peroxidase-conjugated goat anti-mouse IgG antibodies ( $\gamma$-chain specific; Southern Biotech, Birmingham, AL) in PBS containing $0.5 \%$ milk and $0.05 \%$ Tween 20 were incubated for $1 \mathrm{~h}$ at $37{ }^{\circ} \mathrm{C}$. Plates were washed and developed with TMB Microwell Peroxidase Substrate (KPL,
Gaithersburg, MD). The reaction was stopped using $0.5 \mathrm{M}$ sulfuric acid. The absorbance was measured at $450 \mathrm{~nm}$ in a microplate reader (Tecan Sunrise, Grödig, Austria).

\section{Anti-hexa-His tag capture ELISA}

96-well Nunc-Immuno Maxisorp plates (Thermo Scientific, Roskilde, Denmark) were coated overnight at $4{ }^{\circ} \mathrm{C}$ in a wet chamber with $50 \mu \mathrm{l}$ PBS containing anti-hexa-His tag antibodies at a concentration of $5 \mu \mathrm{g} / \mathrm{ml}$. All following steps were carried out on the next day and at room temperature. After washing with distilled $\mathrm{H}_{2} \mathrm{O}$ containing $0.01 \%$ Tween 20, wells were blocked with $5 \%$ non-fat dry milk in PBS for $2 \mathrm{~h}$. Subsequently, wells were incubated with D1NS1-HEK - D4NS1-HEK cell whole protein lysates diluted in PBS for $2 \mathrm{~h}$. After another washing step, wells were incubated with biotinylated anti-NS1 mAbs at a concentration of $5 \mu \mathrm{g} / \mathrm{ml}$ diluted in PBS containing $0.5 \%$ milk for $2 \mathrm{~h}$. Wells were washed and incubated with horseradish peroxidase-conjugated Streptavidin in PBS containing $0.5 \%$ milk for $1 \mathrm{~h}$. Plates were washed and developed with TMB Microwell Peroxidase Substrate (KPL, Gaithersburg, MD). The reaction was stopped using $0.5 \mathrm{M}$ sulfuric acid. The absorbance was measured at $450 \mathrm{~nm}$ in a microplate reader (Tecan Sunrise, Grödig, Austria).

\section{Commercial DENV NS1 capture ELISA}

Lysates of VeroE6 cells infected with different DENV serotypes were analyzed for their NS1 content by a DENV NS1-specific capture ELISA (EUROIMMUN, Luzern, Switzerland) according to the manufacturer's instructions. The three calibrator samples supplied with the kit were applied to assess relative units of the NS1 protein present in the samples.

\section{Results}

Expression of D1NS1 and D4NS1 on the surface of transfected HEK 293F cells

After transfection of HEK cells with the NS1 fusion protein-encoding plasmids pcDNA3.1_BVM_D1NS1_ FLAG_TM_HIS or pcDNA3.1_BVM_D4NS1_FLAG_ TM_HIS, the expression of D1NS1 and D4NS1 by the transfectants was analyzed. No elevated expression levels of a protein corresponding in size to the NS1 protein was visible upon aqua-staining of the SDS-PAGE separated total proteins of D1NS1-HEK and D4NS1-HEK cell lysates when compared to a lysate of untransfected HEK cells (Fig. 1a). However, Western blot analysis with antihexa-His tag antibodies (Fig. 1b) confirmed the specific expression of the NS1 fusion proteins (a band at the expected size for the monomer NS1 of 46-55 kDa [20]) by the transfectants. In combination with Western blot analysis with anti-tubulin antibodies serving as a loading control (Fig. 1c), these analyses revealed no significant difference between expression levels of D1NS1 and D4NS1. 

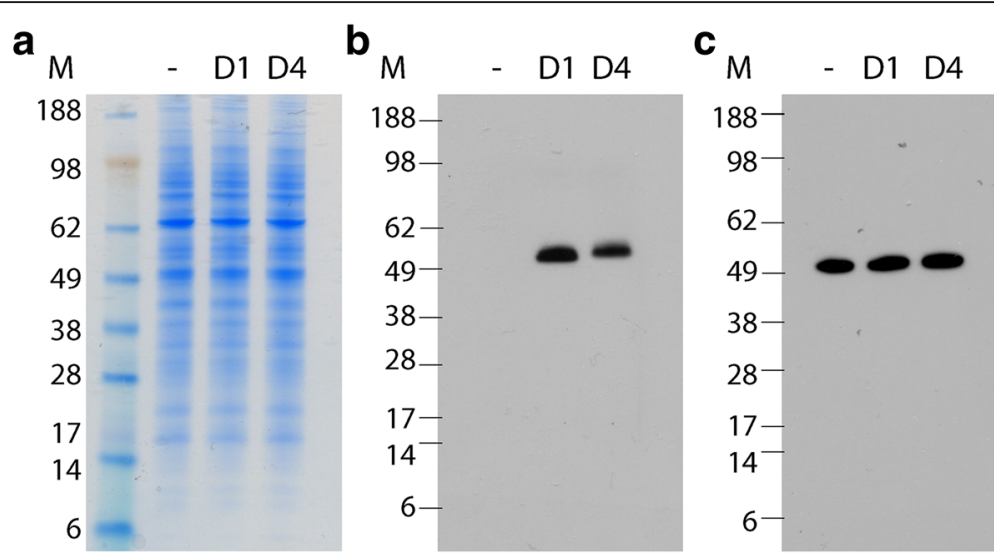

Fig. 1 Expression of D1NS1 and D4NS1 by transfected HEK cells. HEK cell lysates were analyzed by aqua-staining and Western blot analysis after separation on NuPAGE Novex 4-12\% Bis-Tris Gels under reducing conditions (addition of reducing agent and heating of the samples). While aqua-staining of lysates prepared from HEK-derived cell lines expressing D1NS1 (D1) and D4NS1 (D4) showed no additional band of the predicted size of the NS1 proteins, as compared to the lysates of untransfected HEK cells $(-)(\mathbf{a})$, Western blot analysis using anti-hexa-His tag antibodies confirmed the expression of D1NS1 and D4NS1 by the HEK cells (b). Western blotting using anti-tubulin antibodies was performed as a control for the amount of cellular proteins of the untransfected and transfected HEK cell lysates loaded on the gels (c). $M=$ molecular weight marker in $\mathrm{kDa}$

IFA of D1NS1-HEK, D4NS1-HEK and untransfected HEK cells with anti-hexa-His tag antibodies demonstrated the cell surface localization of the expressed NS1 fusion proteins with similar staining intensities for D1NS1 and D4NS1 (Fig. 2).

\section{Development of DENV NS1-specific antibody responses in} mice by immunization with transfected HEK cells

Immunization of mice with either D1NS1-HEK or D4NS1-HEK cells resulted in the development of humoral immune responses specific for the NS1 proteins (Fig. 3).
Sera of mice immunized with D1NS1-HEK contained higher titers of antibodies against recD1NS1 (Fig. 3a) than against recD4NS1 (Fig. 3c). In contrast, similar antibody titers to both recD1NS1 and recD4NS1 were detected in mice that were immunized with D4NS1-HEK cells (Fig. 3b and d). Individual mouse antisera of the D1NS1-HEK and D4NS1-HEK mouse groups contained similar levels of antibodies to the NS1 target protein. All mouse antisera showed only very low anti-hexa-His tag antibody levels, as determined by ELISA of the sera on an unrelated hexaHis tagged protein (MUL_3720) (Fig. 3e and f).

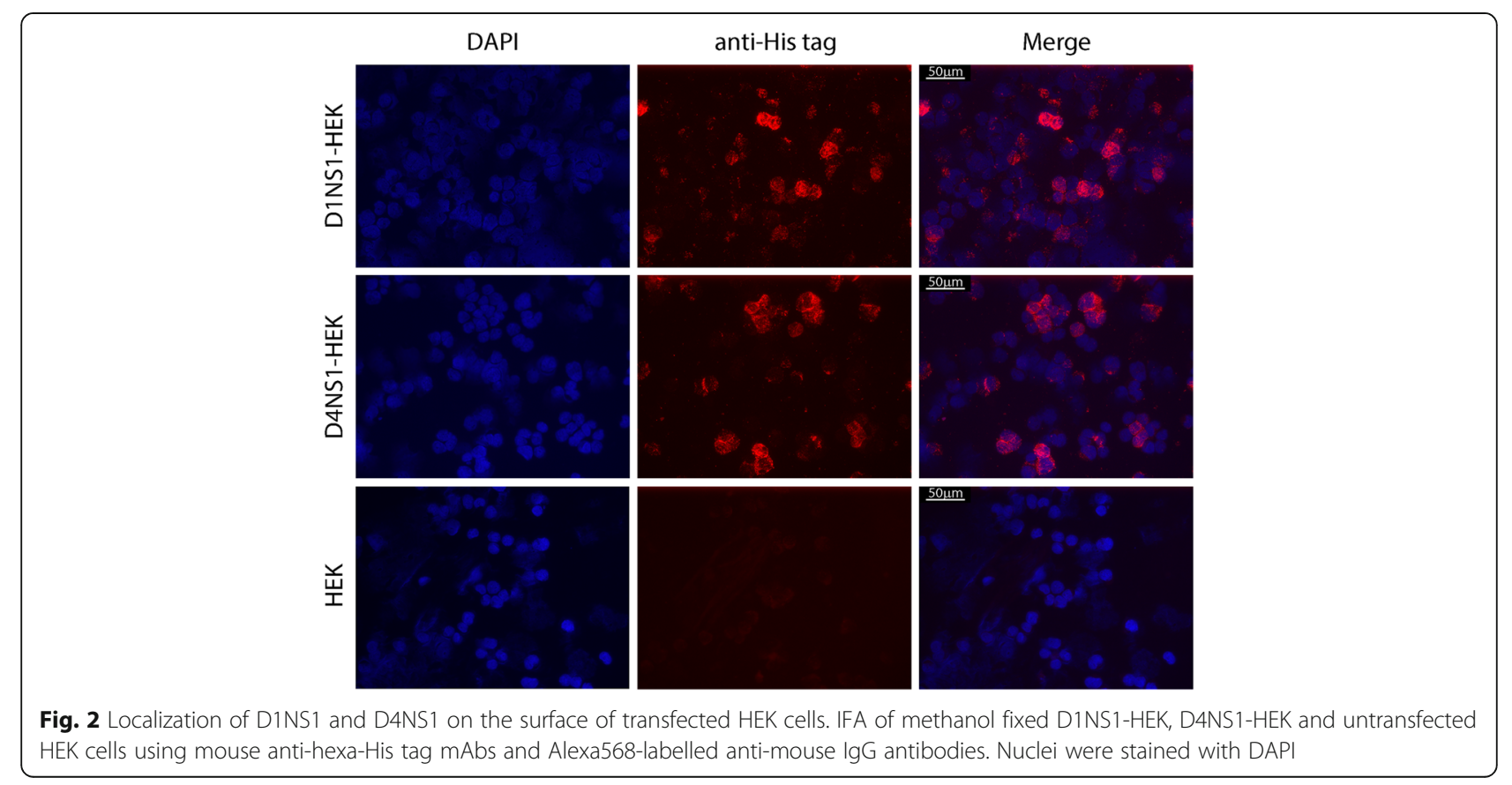




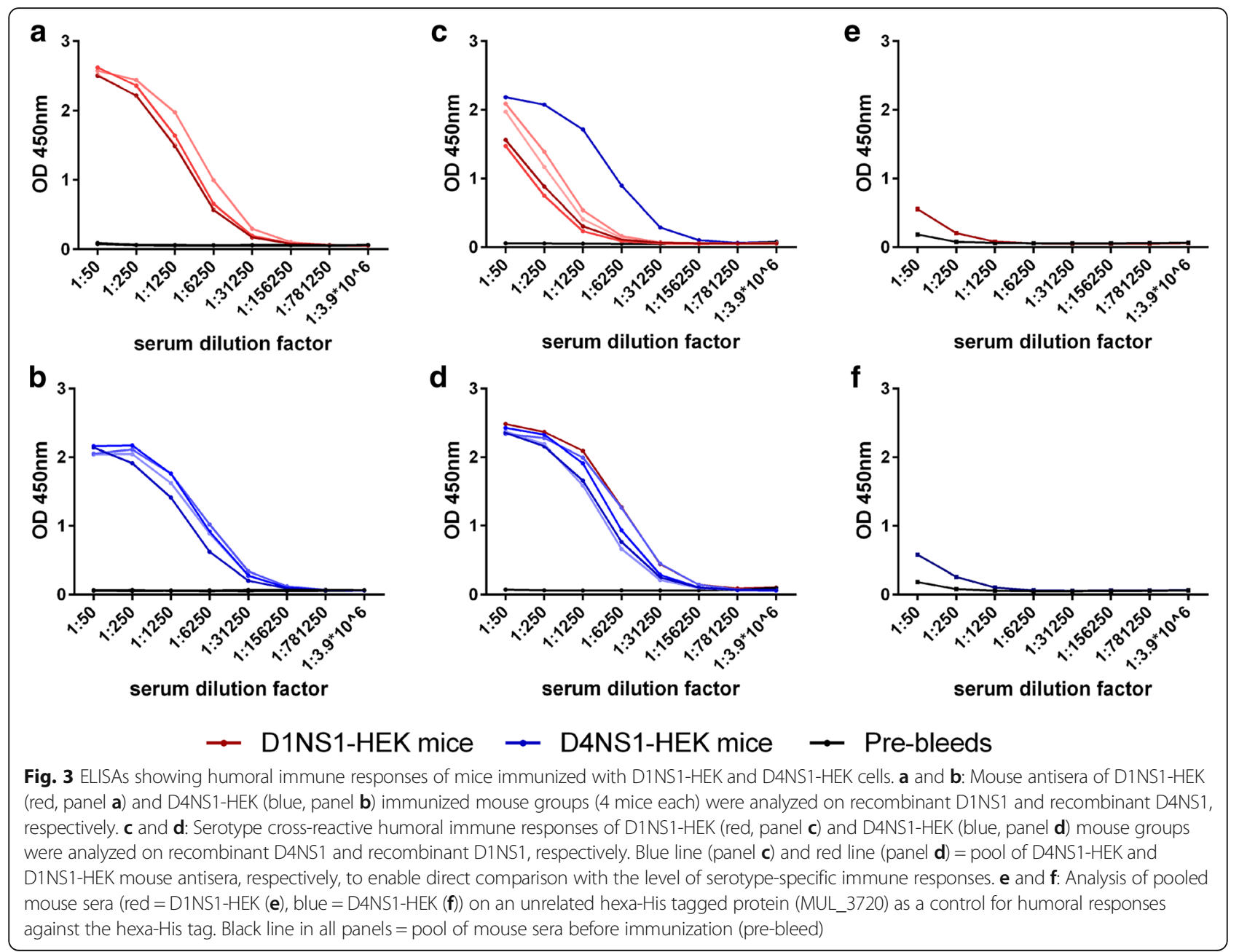

Table 1 Characteristics and reactivities of the generated anti-NS1 mAbs

\begin{tabular}{|c|c|c|c|c|c|c|c|c|c|c|c|}
\hline \multirow[b]{3}{*}{$\mathrm{mAb}$} & \multirow[b]{3}{*}{ Isotype } & \multirow{2}{*}{\multicolumn{2}{|c|}{$\frac{\text { IFA }}{\text { Transfected HEK cells }}$}} & \multirow{2}{*}{\multicolumn{4}{|c|}{$\frac{\text { ELISA }}{\text { Transfected HEK cell lysates }}$}} & \multicolumn{4}{|c|}{ Western blot analysis } \\
\hline & & & & & & & & \multicolumn{4}{|c|}{ DENV-infected Vero E6 cell lysates } \\
\hline & & D1 & D4 & D1 & D2 & D3 & D4 & D1 & D2 & D3 & D4 \\
\hline NR1.1 & $\lg \mathrm{G} 1, \mathrm{k}$ & + & - & + & - & - & - & + & - & - & - \\
\hline NR1.2 & $\lg G 1, \mathrm{k}$ & + & - & + & - & - & - & + & - & + & - \\
\hline NR1.3 & $\lg \mathrm{l} 1, \mathrm{k}$ & + & - & + & - & - & - & + & - & - & - \\
\hline NR1.4 & $\operatorname{lgG} 2 \mathrm{a}, \mathrm{k}$ & + & + & + & + & + & - & + & + & + & - \\
\hline NR1.5 & $\lg G 2 \mathrm{a}, \mathrm{k}$ & + & + & + & - & - & + & + & - & - & - \\
\hline NR1.6 & $\lg G 1, \mathrm{k}$ & + & - & + & - & - & - & + & - & - & - \\
\hline NR1.7 & $\lg G 2 \mathrm{a}, \mathrm{k}$ & + & - & + & - & - & - & + & - & - & - \\
\hline NR1.8 & $\lg G 2 \mathrm{~b}, \mathrm{k}$ & + & + & + & - & + & + & + & + & + & + \\
\hline NR4.1 & $\lg G 2 \mathrm{a}, \mathrm{k}$ & - & + & - & - & - & + & - & - & - & + \\
\hline NR4.2 & $\lg G 2 \mathrm{a}, \mathrm{k}$ & - & + & - & - & - & + & - & - & - & + \\
\hline NR4.3 & $\lg G 1, \mathrm{k}$ & + & + & - & - & - & + & - & - & - & + \\
\hline NR4.4 & $\lg G 2 \mathrm{a}, \mathrm{k}$ & - & + & - & - & - & + & - & - & - & + \\
\hline
\end{tabular}

The different recognition patterns of the 12 generated anti-NS1 mAbs as determined by i) IFA on the D1NS1 and D4NS1 transfected HEK cells, ii) ELISA on whole protein lysates of D1NS1-HEK - D4NS1-HEK cells and iii) Western blot analysis on whole protein lysates of DENV-infected VeroE6 cells are summarized 
Generation and characterization of anti-DENV NS1 mAbs Fusion of spleen cells obtained from mice immunized with D1NS1-HEK or D4NS1-HEK cells with PAI myeloma cells enabled the generation of anti-NS1 antibodyproducing hybridoma cell lines. In order to identify cell lines that produce IgG specific for NS1, hybridoma supernatants were screened by ELISA on recD1NS1 or recD4NS1, respectively. Supernatants from ELISA-positive wells were subsequently tested by IFA on D1NS1-HEK and D4NS1-HEK cells. IFA on HEK cells transfected with an unrelated hexa-His tagged protein served as control to identify those hybridoma cell lines that generated NS1-specific antibodies. Screening of D1NS1-HEK and D4NS1-HEK mouse spleen cell fusions yielded eight and four anti-DENV NS1 IgG-producing hybridoma cell lines, respectively. These cell lines were cloned by limiting dilution to obtain hybridoma clones producing the mAbs NR1.1 - NR1.8 and NR4.1 - NR4.4, respectively. Five mAbs were of the $\operatorname{IgG} 1(\mathrm{k})$, six of the $\operatorname{IgG} 2 \mathrm{a}(\mathrm{\kappa})$ and one of the IgG2b(k) IgG subclass (Table 1). IFA with these mAbs on the D1NS1 and D4NS1 transfectants revealed that five specifically recognized D1NS1-HEK but not D4NS1-HEK cells, three only reacted with D4NS1-HEK cells and the remaining four were cross-reactive with both transfectants (Table 1, Fig. 4).

In order to test serotype-specificity of the generated mAbs on all four DENV serotypes, analyses were extended to HEK cells expressing D2NS1 and D3NS1 (Additional file 2: Figure S2). When serotype specificity was tested by ELISA using anti-hexa-His tag antibodies as common capturing reagent for all four hexa-His tagged NS1 fusion proteins from HEK cell lysates, different recognition patterns were observed for the 12 antiNS1 mAbs used as biotinylated detecting reagents in ELISA. While five of the eight mAbs raised against D1NS1 (NR1.1, NR1.2, NR1.3, NR1.6 and NR1.7) specifically recognized only D1NS1, three (NR1.4, NR1.5 and NR1.8) also cross-reacted with the NS1 protein of at least one other serotype. In contrast, all four mAbs raised against D4NS1 (NR4.1 - NR4.4) reacted only with D4NS1 in ELISA (Table 1, Fig. 5).

\section{Detection of virus material derived from DENV-infected VeroE6 cells by the generated mAbs}

Cross-reactivity of the generated mAbs with virusinfected cells was analyzed by Western blot analysis with lysates of VeroE6 cells infected with the four different DENV serotypes (Fig. 6). Results were nearly identical to the ELISA analysis of all four hexa-His tagged NS1 fusion proteins from HEK cell lysates with three exceptions: i) the Western blot cross-reactive mAb NR1.8 did not react with the D2NS1 protein in the ELISA, ii) $\mathrm{mAb}$ NR1.2 did recognize D3NS1 in virus material, but not the NS1 protein present in D3NS1-HEK cell lysates and

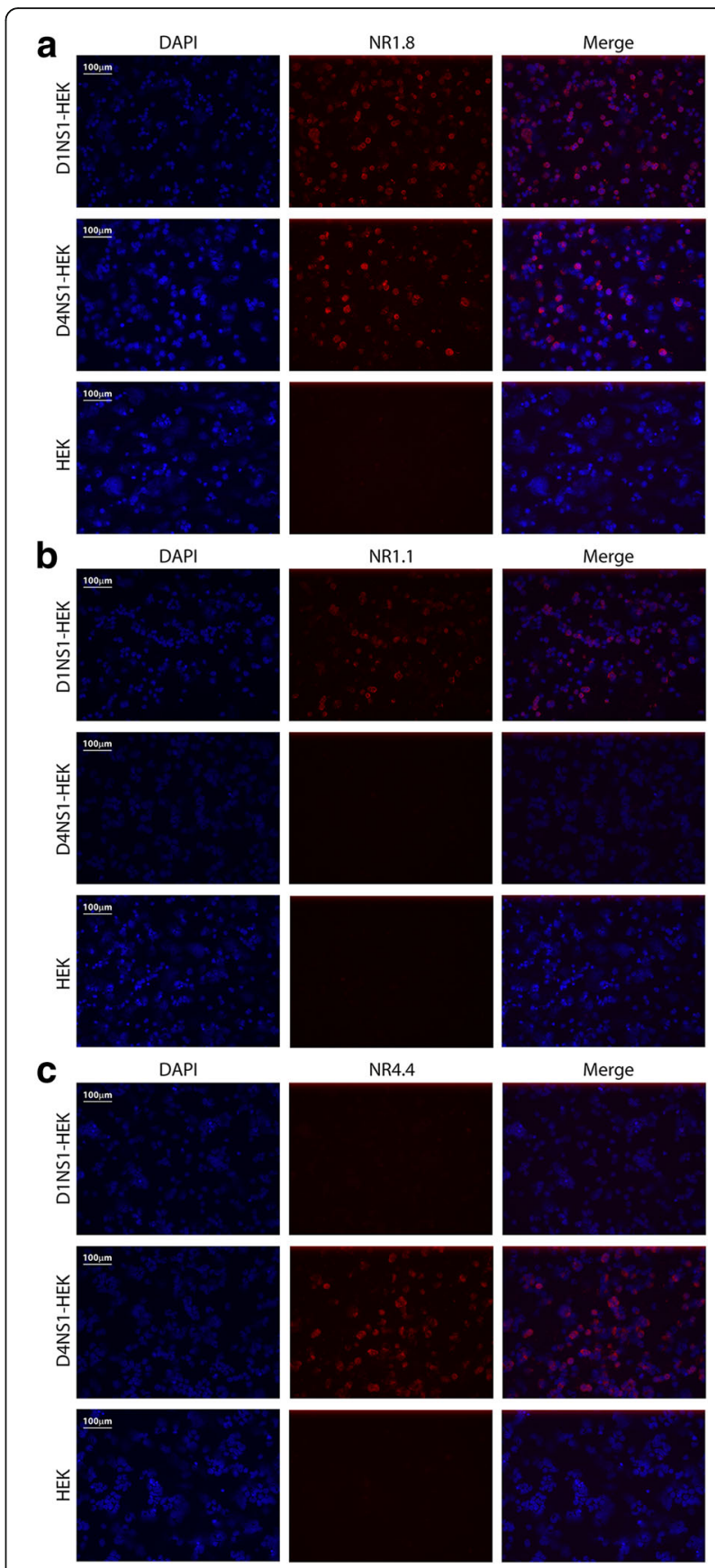

Fig. 4 IFA of selected generated anti-NS1 mAbs on HEK cells. IFA of methanol fixed D1NS1-HEK, D4NS1-HEK and untransfected HEK cells after staining with selected generated anti-NS1 mAbs and Alexa568labelled anti-mouse lgG antibodies. a: D1NS1 and D4NS1 serotype cross-reactive mAb NR1.8. $\mathbf{b}$ : D1NS1 serotype-specific mAb NR1.1. c: D4NS1 serotype-specific mAb NR4.4. Nuclei were stained with DAPI

iii) mAb NR1.5 recognized NS1 in D4NS1-HEK cell lysates, but not the one present in the virus-infected cell lysates. Analysis with lysates of the virus-infected cells showed that mAb NR1.8 is cross-reactive with all four DENV serotypes. Five of the mAbs specifically reacted 


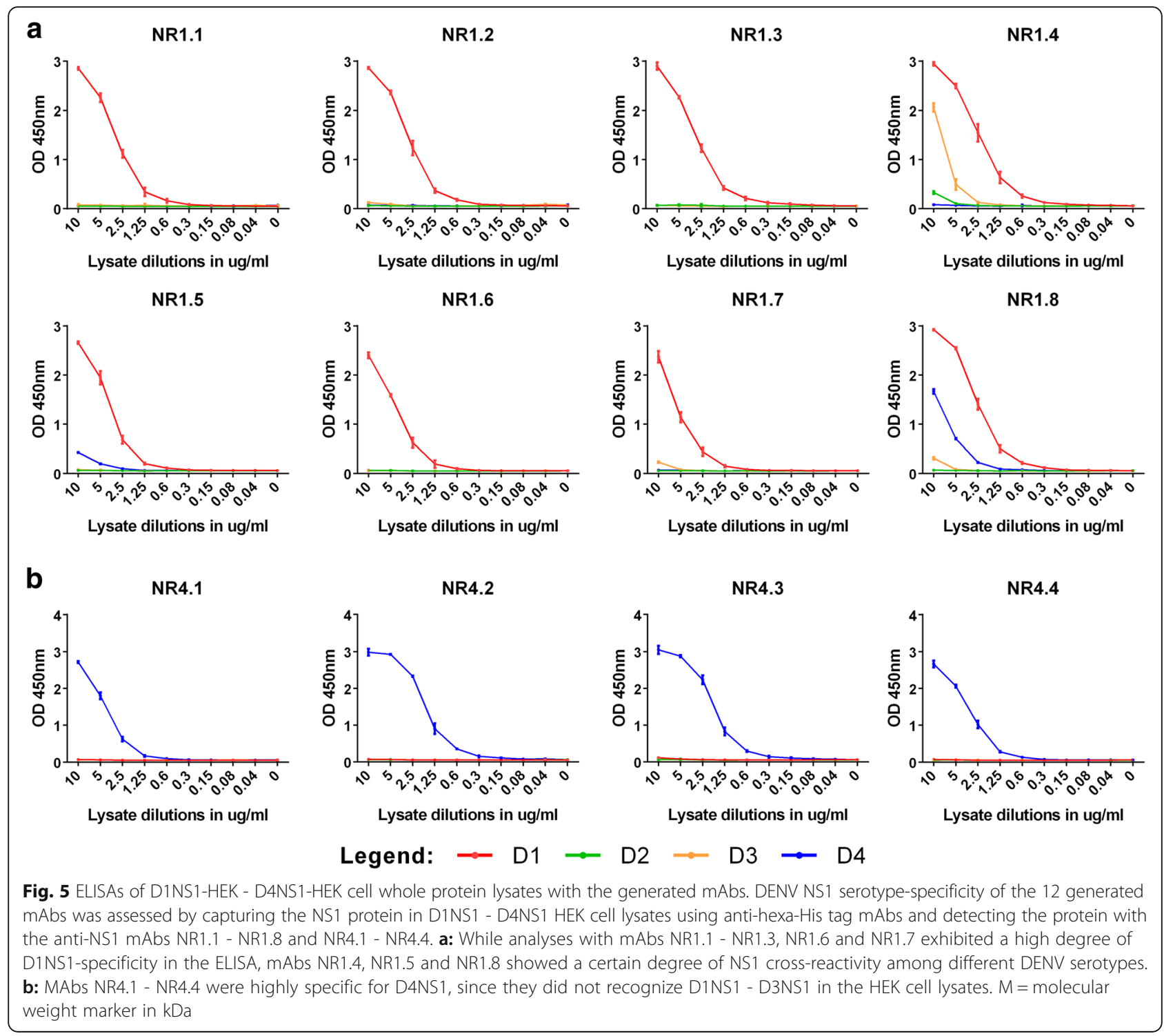

with D1NS1 and four recognized D4NS1 only. One of the mAbs cross-reacted with D1NS1 - D3NS1, but did not recognize D4NS1 and one reacted with D1NS1 and D3NS1 (Fig. 6, Table 1).

\section{Discussion}

In the present study, we describe an efficient and costeffective strategy to rapidly generate mouse mAbs against native viral proteins. The methodology used is based on the immunization of mice with HEK cells presenting the native target proteins on their surface. Expression of the proteins on the HEK cell surface is achieved by hijacking the HEK cell's synthetic protein machinery upon transfection of the cells with a plasmid encoding the target antigen as fusion protein connected to a transmembrane domain [22]. The use of HEK cells as a tool for the expression of native proteins has several advantages. For one, HEK cells are easy to grow in culture, can efficiently be transfected with commercially available transfection reagents, and are also capable of expressing large amounts of recombinant proteins [25]. Moreover, mammalian cell cultures - as opposed to prokaryotic or yeast expression systems - represent an optimal tool for the production of viral proteins (particularly glycoproteins [26]), since protein folding and post-translational modifications closely approximate those occurring in the infected mammalian hosts [27]. The immunization of mice with antigens hooked to the surface of HEK cells has the further advantages that neither elaborate production/ processing of recombinant antigen nor addition of adjuvant - that both may negatively affect protein folding are required.

Here we used this novel approach to rapidly generate panels of mAbs against the DENV NS1 protein. 


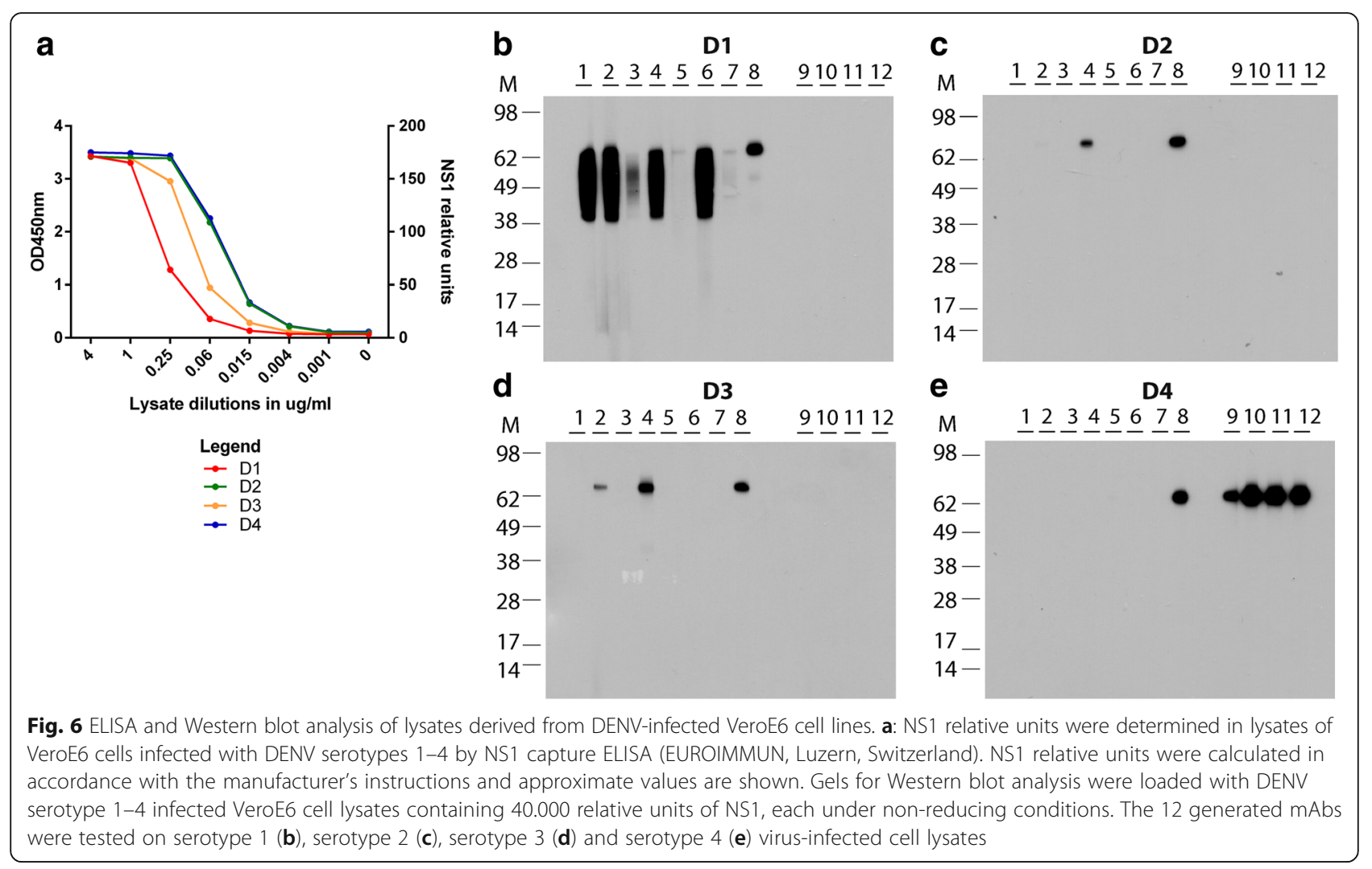

Immunization of mice with D1NS1-HEK and D4NS1HEK cells, whose viability was preserved by storage at $-80{ }^{\circ} \mathrm{C}$ in freezing medium, and subsequent production of hybridoma clones yielded D1NS1 - and D4NS1 serotype-specific as well as DENV NS1 serotype crossreactive mAbs. In view of the $30 \%$ variability present among sequences of the four DENV serotypes at the amino acid level (Additional file 1: Figure S1), it was expected that mice immunized with one serotype will develop both serotype-specific and serotype crossreactive humoral immune responses. In this study a first selection of the desired mAbs was achieved by high-throughput ELISA screening of the hybridoma cell supernatants on small amounts of commercially available DENV D1NS1 - D4NS1 recombinant proteins. However, in order to completely circumvent the use of recombinant proteins, lower throughput screening can also be performed by IFA on HEK cells transfected with the target protein or by Western blot analysis on lysates of transfected HEK cells. These assays should be performed in parallel with tests using material from HEK cells transfected with an unrelated hexa-His tagged fusion protein to exclude hybridomas producing HEK cell and hexa-His tag -specific antibodies from further analysis. Seven of the identified mAbs (the D1NS1-specific NR1.1, NR1.3, NR1.6 and NR1.7 and the D4NS1-specific NR4.1, NR4.2 and NR4.4) were serotype-specific in all applied assay formats, while one mAb (NR1.8) reacted with the NS1 protein of each of the four serotypes in all but one (ELISA on D2NS1) of the assays. The other four mAbs (NR1.2, NR1.4, NR1.5 and NR4.3) reacted with the NS1 protein of at least two, but not all of the four DENV serotypes (Table 1). Discrepancies in recognition patterns might be explained by steric differences (hindrances) in binding of the mAbs in different assay formats. Western blot analysis of DENV-infected cell lysates confirmed that the generated mAbs can be used to detect the endogenous NS1 protein in DENV-infected biological samples. Differences in recognition patterns might also be explained by sub-serotype specificity of the generated mAbs.

The progressive and ubiquitous threat of newly emerging and re-emerging viral diseases necessitates the development of tools for their prevention, detection and control. In the past decades mAbs have been increasingly used for the development of vaccines, diagnostics and therapeutics as well as for research on the epidemiology of pathogens. In this study we were able to prove our approach of rapidly generating panels of mAbs against native viral proteins. The envisaged production of serotype and sub-serotype variant specific anti-NS1 mAbs against all DENV serotypes will provide various prospects for their application. Antigen capture assays for diagnostic purposes may be developed by using a broadly cross-reactive 
capturing $\mathrm{mAb}$ in combination with different serotypespecific detection mAbs. Variant specific mAbs may be used as tools to study the epidemiology of DENV isolates based on their antigenic diversity [12].

\section{Conclusions}

Our cell-based system of immunizing mice with HEK cells presenting native viral proteins followed by hybridoma selection provides a highly efficient tool to rapidly obtain mAbs against native viral proteins that can be used for various downstream applications. The strategy can easily be extended to a broad range of viral proteins.

\section{Additional files}

Additional file 1: Figure S1. Dengue virus NS1 protein sequences. Sequence alignment of the D1NS1 - D4NS1 protein sequences expressed by the transfected HEK cells showing $30 \%$ sequence variability. Identical amino acid positions among the four serotypes are highlighted in grey. (TIF $2689 \mathrm{~kb}$ )

Additional file 2: Figure S2. Expression of D2NS1 and D3NS1 by transfected HEK cells. While aqua staining of lysates prepared from HEK-derived cell lines expressing D2NS1 (D2) and D3NS1 (D3) showed no specific band for the NS1 protein as compared to the lysates of untransfected HEK cells $(-)(\mathbf{a})$, Western blot analysis using anti-hexa-His tag antibodies confirmed the expression of D2NS1 and D3NS1 by the HEK cells (b). Western blotting using anti-tubulin antibodies was performed as a control for the amount of untransfected and transfected HEK cell lysates loaded on the gels (c). $M=$ molecular weight marker in $\mathrm{kDa}$. (TIF $3371 \mathrm{~kb}$ )

\section{Acknowledgements}

We thank Christoph Schäfer for the provision of the Euroimmun DENV NS1 capture ELISA kits and Marcello Foggetta for cell transfections.

\section{Funding}

The laboratory work was financed by funds of the Swiss TPH. Carlos Augusto Pinho-Nascimento was supported by a stipend of the Brazilian National Council of Technological and Scientific Development (CNPq). The concept of the study was developed in cooperation with the Unisciencia foundation.

\section{Availability of data and material}

All data generated during this study are included in this published article and its additional files.

\section{Authors' contributions}

NR was a main contributor in the generation of anti-DENV NS1 mAbs, performance of experiments and analysis of data. CAP participated in the study design and performance of experiments. AR and HR provided the DENV material analyzed in this study. PF was a major contributor in the experimental design of mAb generation. MT performed the mouse immunizations. MTB, HM and TJ participated in the design of the study. GP was a major contributor in study and experimental design and revised the manuscript. KR was a major contributor in study and experimental design and performance of experiments and drafted the manuscript. All authors have read and approved the final manuscript.

\section{Competing interests}

The authors declare that they have no competing interests.

\section{Consent for publication}

Not applicable.

\section{Ethics approval and consent to participate}

Immunization of Naval Medical Research Institute (NMRI) mice was performed in strict accordance with the rules and regulations for the protection of animal rights of the Swiss Federal Food Safety and Veterinary Office. Experiments were approved by the animal welfare committee of the Canton of Basel (authorization number 2375).

\section{Author details}

${ }^{1}$ Swiss Tropical and Public Health Institute, Molecular Immunology, Basel, Switzerland. ${ }^{2}$ University of Basel, Basel, Switzerland. ${ }^{3}$ Biology Institute, Fluminense Federal University, Laboratory of Molecular Virology, Niterói, Brazil. ${ }^{4}$ Department of Infectious Diseases, University of Heidelberg, Molecular Virology, Heidelberg, Germany. ${ }^{5}$ Oswaldo Cruz Institute, Fiocruz, Laboratory of comparative and environmental Virology, Rio de Janeiro, Brazil. 'Section Clinical Tropical Medicine, Department of Infectious Diseases, Heidelberg University Hospital, Heidelberg, Germany.

Received: 1 August 2016 Accepted: 1 November 2016 Published online: 22 November 2016

\section{References}

1. Cao-Lormeau VM, Musso D. Emerging arboviruses in the pacific. Lancet. 2014:384(9954):1571-2.

2. Guzman A, Isturiz RE. Update on the global spread of dengue. Int J Antimicrob Agents. 2010;36 Suppl 1:S40-42.

3. Murray NE, Quam MB, Wilder-Smith A. Epidemiology of dengue: past, present and future prospects. Clin Epidemiol. 2013;5:299-309.

4. Weaver SC, Lecuit M. Chikungunya virus and the global spread of a mosquito-borne disease. N Engl J Med. 2015:372(13):1231-9.

5. Musso D, Cao-Lormeau VM, Gubler DJ. Zika virus: following the path of dengue and chikungunya? Lancet. 2015;386(9990):243-4.

6. Duffy MR, Chen TH, Hancock WT, Powers AM, Kool JL, Lanciotti RS, Pretrick M, Marfel M, Holzbauer S, Dubray C, et al. Zika virus outbreak on Yap Island, Federated States of Micronesia. N Engl J Med. 2009;360(24):2536-43.

7. Brasil $P$, Calvet GA, Siqueira AM, Wakimoto M, de Sequeira PC, Nobre A, Quintana Mde S, Mendonca MC, Lupi O, de Souza RV, et al. Zika virus outbreak in Rio de Janeiro, Brazil: clinical characterization, epidemiological and virological aspects. PLoS Negl Trop Dis. 2016;10(4):e0004636.

8. loos S, Mallet HP, Leparc Goffart I, Gauthier V, Cardoso T, Herida M. Current Zika virus epidemiology and recent epidemics. Med Mal Infect. 2014;44(7):302-7.

9. Bhatt S, Gething PW, Brady OJ, Messina JP, Farlow AW, Moyes CL, Drake JM, Brownstein JS, Hoen AG, Sankoh O, et al. The global distribution and burden of dengue. Nature. 2013;496(7446):504-7.

10. Guzman MG, Harris E. Dengue. Lancet. 2015;385(9966):453-65.

11. OhAinle M, Balmaseda A, Macalalad AR, Tellez Y, Zody MC, Saborio S, Nunez A, Lennon NJ, Birren BW, Gordon A, et al. Dynamics of dengue disease severity determined by the interplay between viral genetics and serotypespecific immunity. Sci Transl Med. 2011;3(114):114ra128.

12. Katzelnick LC, Fonville JM, Gromowski GD, Bustos Arriaga J, Green A, James SL, Lau L, Montoya M, Wang C, VanBlargan LA, et al. Dengue viruses cluster antigenically but not as discrete serotypes. Science. 2015;349(6254):1338-43.

13. Kohler $\mathrm{G}$, Milstein C. Continuous cultures of fused cells secreting antibody of predefined specificity. Nature. 1975;256(5517):495-7.

14. Siddiqui MZ. Monoclonal antibodies as diagnostics; an appraisal. Indian J Pharm Sci. 2010;72(1):12-7.

15. Dimitrov DS. Therapeutic antibodies, vaccines and antibodyomes. MAbs 2010;2(3):347-56.

16. Buss NA, Henderson SJ, McFarlane M, Shenton JM, de Haan L. Monoclonal antibody therapeutics: history and future. Curr Opin Pharmacol. 2012; 12(5):615-22.

17. Sorensen HP. Towards universal systems for recombinant gene expression. Microb Cell Fact. 2010:9:27.

18. Portolano N, Watson PJ, Fairall L, Millard CJ, Milano CP, Song Y, Cowley SM, Schwabe JW. Recombinant protein expression for structural biology in HEK 293 F suspension cells: a novel and accessible approach. J Vis Exp. 2014;92:e51897.

19. Lindenbach $B D$, Rice CM. Molecular biology of flaviviruses. Adv Virus Res. 2003:59:23-61.

20. Muller DA, Young PR. The flavivirus NS1 protein: molecular and structural biology, immunology, role in pathogenesis and application as a diagnostic biomarker. Antiviral Res. 2013:98(2):192-208.

21. Alcon S, Talarmin A, Debruyne M, Falconar A, Deubel V, Flamand M. Enzyme-linked immunosorbent assay specific to dengue virus type 1 nonstructural protein NS1 reveals circulation of the antigen in the blood 
during the acute phase of disease in patients experiencing primary or secondary infections. J Clin Microbiol. 2002;40(2):376-81.

22. Dreyer AM, Beauchamp J, Matile H, Pluschke G. An efficient system to generate monoclonal antibodies against membrane-associated proteins by immunisation with antigen-expressing mammalian cells. BMC Biotechnol. 2010;10:87.

23. Pluschke G, Joss A, Marfurt J, Daubenberger C, Kashala O, Zwickl M, Stief A, Sansig G, Schlapfer B, Linkert S, et al. Generation of chimeric monoclonal antibodies from mice that carry human immunoglobulin Cgamma1 heavy of Ckappa light chain gene segments. I Immunol Methods. 1998;215(1-2):27-37.

24. Dreyer A, Roltgen K, Dangy JP, Ruf MT, Scherr N, Bolz M, Tobias NJ, Moes C, Vettiger A, Stinear TP, et al. Identification of the Mycobacterium ulcerans protein MUL_3720 as a promising target for the development of a diagnostic test for Buruli ulcer. PLoS Negl Trop Dis. 2015;9(2):e0003477.

25. Thomas P. Smart TG. HEK293 cell line: a vehicle for the expression of recombinant proteins. J Pharmacol Toxicol Methods. 2005;51(3):187-200.

26. Aricescu AR, Owens RJ. Expression of recombinant glycoproteins in mammalian cells: towards an integrative approach to structural biology. Curr Opin Struct Biol. 2013;23(3):345-56.

27. Fields BN, Knipe DM, Griffin DE. Fields virology. 6th ed. 2013.

\section{Submit your next manuscript to BioMed Central and we will help you at every step:}

- We accept pre-submission inquiries

- Our selector tool helps you to find the most relevant journal

- We provide round the clock customer support

- Convenient online submission

- Thorough peer review

- Inclusion in PubMed and all major indexing services

- Maximum visibility for your research

Submit your manuscript at www.biomedcentral.com/submit 